\title{
Asymmetric Delay in Evolutionary Games
}

\author{
Hamidou Tembine * , Eitan Altman ${ }^{+}$and Rachid El-Azouzi * \\ * LIA-CERI, University of Avignon, 339, chemin des Meinajaries Agroparc \\ B.P. 1228, 84911 Avignon Cedex 9, France \\ \{hamidou.tembine,rachid.elazouzi\}@univ-avignon.fr \\ + INRIA, 2004 Route des Lucioles, \\ B.P.93, 06902 Sophia-Antipolis Cedex, France \\ eitan.altman@sophia.inria.fr
}

\begin{abstract}
We study the effect of the time delays on the convergence of replicator dynamics to the evolutionary stable strategies in a symmetric evolutionary game in which each pure strategy is associated with its own delay. We compare the stability region to that obtained when all strategies have the same delay. Multiple Access Game and Hawk and Dove Game are studied.
\end{abstract}

\section{Keywords}

hawk and dove game, multiple access game, evolutionary stable strategy, delay differential equation, replicator dynamics.

\section{INTRODUCTION}

Evolutionary game theory is developed by biologists for predicting population dynamics in interaction. It differs from classical game theory by (i) its focusing on the evolution dynamics of the fraction of members of the population that use a given strategy, and (2) in the notion of Evolutionary Stable Strategy (ESS, [27]) which includes robustness against a deviation of a whole (possibly small) fraction of the population who may wish to deviate (This is in contrast with the standard Nash equilibrium that only incorporates robustness against deviation of a single user).

Recently, however, evolutionary game theory has become of increased interest to social scientists [13]. In computer science, evolutionary game theory is appearing, some examples of applications can be found in multiple access protocols [6], multihoming[26] and resources competition in the Internet[35].

We assume there is a time delay between the moment that a strategy is used till the player feels the impact of its action (i.e. till it receives the corresponding fitness ). Time delay can represent a time scale much slower than the physical delays $[18,3]$. In a computer network, the evolution time scale could be of the order of months or years and could

Permission to make digital or hard copies of all or part of this work for personal or classroom use is granted without fee provided that copies are not made or distributed for profit or commercial advantage and that copies bear this notice and the full citation on the first page. To copy otherwise, to republish, to post on servers or to redistribute to lists, requires prior specific permission and/or a fee.

Valuetools '07, October 23-25, 2007, Nantes, France.

Copyright 2007 ICST 978-1-59593-819-00-4. apply to the evolution of services or of protocols, whereas the physical delays can be of the order of a second [18] or less.

Related work. Delay and its impact on system stability have been studied in many engineering contexts. Much recent work can be found in the area of flow and congestion control $[2,22,18]$. Several studies have considered delays in evolutionary games. Tao and Wang[34] studied the effect of a (symmetric) time delay on the stability of interior stationary points of replicator dynamics[31]. They considered a two-player game (i.e the interactions between populations of strategies occur between many encounters of pairs of players) with two strategies and a unique asymptotically stable interior stationary point and they showed that the mixed ESS can be an unstable equilibrium state of the population if the delay is large. A similar result is proved by Alboszta and Miękisz [1] in the discrete replicator dynamics of the social model.

Our goal is to study the effect of time delays in evolutionary games with one population of users and two strategies. We assume as usual that the interactions between the strategies are manifested through many local interactions between pairs of players. We shall use the standard representations of these as a two players matrix game, each player with two strategies; the expected fitness of a player is computed by using as strategy for the other player the fraction of the population that uses each pure strategy.

We study in particular the case where the delays corresponding to different strategies are not the same. We study the stability as a function of delays. We derive new stability conditions for the replicator dynamics with non-symmetric delays and apply it to the Hawk and Dove game and the Multiple Access Game.

The paper is structured as follows. We first provide in the next section the needed background on delayed evolutionary games. We then study the stability of ESS for the case of $n$ strategies. Next, we investigate the impact of the choice of some parameters in the replicator dynamics on the stability of the system in Section 3 and apply the results to the Multiple Access Game and Hawk and Dove Game. Finally we give some stability condition in games with delay for any integer number $n$ of strategies. We discuss how to use Nyquist stability criterion with the replicator equation.

\section{EVOLUTIONARY GAMES WITH DELAYED DYNAMICS}

We consider a large population of players. We assume that 
there are $n$ pure strategies. A strategy of an individual is a probability distribution over the pure strategies. An equivalent interpretation of strategies is obtained by assuming that individuals choose pure strategies and then the probability distribution represents the fraction of individuals in the population that choose each strategy.

We denote by $A=\left(K a_{i j}\right)_{i, j=1, \ldots, n}$ the payoff matrix where $K$ is a positive parameter (this parameter doesn't change the equilibrium set). The individuals compete through a large number of random pairwise interactions. If an individual uses at time $t$ a strategy $k$ then it receives an expected payoff (fitness) at time $t+\tau_{k}$ where $\tau_{k}$ is the time delay of the strategy $k$.

Let $x$ be the $n$ dimensional vector whose $i$-th element $x_{i}$ is the population share of strategy $i$. Let $f_{i}(x)$ be the expected payoff value of the strategy $i$ when the composition of population is $x$. The payoff of the individual using the strategy $i$ is $F_{i}(x):=R+f_{i}(x)$ where $R$ is the individual's payoff when there is no game (at the initial time). The payoff function $f=\left(f_{i}\right)_{i=1, \ldots, n}$ is given by

$$
f_{i}(x)=e_{i} A x
$$

where $e_{i} i$-th element of the canonical basis of $\mathbb{R}^{n}$. Note that $F$ is affine in $x$. Equivalently, $F_{i}(x)$ can be interpreted as the payoff of a player in the matrix-game using strategy $i$ when it encounters a player with mixed strategy $x$.

\section{ESS}

A state $x^{*}$ is an ESS, if for all $x \neq x^{*}$, there exists some $\epsilon_{x}>0$ such that

$$
x^{*} A\left(\epsilon x+(1-\epsilon) x^{*}\right)>x A\left(\epsilon x+(1-\epsilon) x^{*}\right)
$$

for all $\epsilon \in\left(0, \epsilon_{x}\right)$. That is, $x^{*}$ is ESS if, after mutation, nonmutants are more successful than mutants, in which case mutants cannot invade and will eventually get extinct. The number $\epsilon_{x}$ is called invasion barrier [33]. It is the maximum rate of mutants against which $x^{*}$ is resistant. The conditions to be an ESS can be interpreted in terms of Nash equilibrium in a matrix game. If $x^{*}$ is an ESS then $x^{*}$ is a Nash equilibrium. Equivalently, $x^{*}$ is an ESS if and only if it meets the best reply conditions:

$$
\begin{gathered}
x A x^{*} \leq x^{*} A x^{*}, \forall x, \text { and } \\
x A x^{*}=x^{*} A x^{*} \Rightarrow x A x<x^{*} A x, \quad \forall x \neq x^{*} .
\end{gathered}
$$

\section{Replicator Dynamics}

We introduce here the replicator dynamics which describe the evolution in the population of the various strategies. In the replicator dynamics, the share of a strategy in the population grows at a rate proportional to the difference between the delayed payoff of that strategy and the average delayed payoff of the population. More precisely, consider $n$ strategies and let $x(t)$ be the $n$ dimensional vector whose $i$ th element $x_{i}(t)$ is the population share of strategy $i$ at time $t$. Thus we have $\sum_{i=1}^{n} x_{i}(t)=1$ and $x_{i}(t) \geq 0$. The vector $x$ represents a state of the population.

In the classical replicator dynamics, the payoff of strategy $i$ at time $t$ has an instantaneous impact on the rate of growth of the population size that uses it. An alternative more realistic model for replicator dynamic would have some delay: the payoff acquired at time $t$ will impact the rate of growth $\tau_{i}$ time later.
Suppose that each individual of the population only uses a pure strategy $i=1, \ldots, n$.

Then, the fitness of an individual using the strategy $i$ at time $t$ when the state of the population is $x(t)$ at time $t$ is $F_{i}\left(x\left(t-\tau_{i}\right)\right)$ and the replicator dynamics of $x_{i}(t)$ is given by

$$
\dot{x}_{i}(t)=x_{i}(t)\left[F_{i}\left(x\left(t-\tau_{i}\right)\right)-\bar{F}(x(t))\right], i=1, \ldots, n
$$

where $\bar{F}(x(t))=\sum_{k} x_{k}(t) F_{k}\left(x\left(t-\tau_{k}\right)\right)$ is the expected fitness of the population. The strategies with larger fitness are expected to propagate faster in a population.

There is a close relation between the rest points of the replicator equation and the Nash equilibria given by the (symmetric) finite game called as the folk theorem of evolutionary game theory [8].

We see that the fitness for a player at a given time is determined by the action $i$ taken by the player at that time, as well as by the actions of the population it interacts with, that was taken $\tau_{i}$ units ago. Thus the action of a player at time $t$ determines the delay of the interference from the rest of the population. The following example in the context of wireless communications illustrates this. Consider a transmitter A and a very close receiver B. Transmitter A can decide whether to transmit with frequency $f r_{1}$ or $f r_{2}$. If it transmits with frequency $f r_{i}$ then its signal receives interference at receiver B from transmitters that are located at a distance of $D_{i}$ from the receiver, which translates to a time delay of $\tau_{i}$ (where $i=1,2$ ). Thus indeed, the action of a player determines the delay it will take for actions of other players to be effective.

\section{Convergence to the ESS}

We define the notion of evolutionary stable state in presence of delay in this section. We refer the reader to $[24,34,5$, 16] for these notions without delays. We denote by $\tau$ the maximum of the delays $\tau_{j}, j=1, \ldots, n$.

Definitions 1. 1. The state $x^{*}$ is stationary state (or rest point, see [14]) of the differential equation (1) if it is a critical point i.e the right side of (1) is zero at $x^{*}$. This condition becomes $\forall i, x_{i}^{*}=0$ or $\sum_{j=1}^{n} a_{i j} x_{j}^{*}=$ $\sum_{k} \sum_{l} a_{k l} x_{k}^{*} x_{l}^{*}$

2. $x^{*}$ is stable if it is a stationary point with the property that for every neighborhood $\mathcal{V}$ of $x^{*}$, there exists a neighborhood $\mathcal{U} \subset \mathcal{V}$ with the property that if $x(t) \in \mathcal{U}$ for $t \in(-\tau, 0)$ then $x(t) \in \mathcal{U}$ for all $t>0$.

3. $x^{*}$ is asymptotically stable if it is stable and there exists a neighborhood $\mathcal{W}$ of $x^{*}$ such that $x(t) \in \mathcal{W}$ for all $t \in(-\tau, 0)$ implies $\lim _{t \rightarrow+\infty} x(t)=x^{*}$

4. $x^{*}$ is exponentially stable if it is stable and there exists $t_{0}, L, \eta>0$ such that $\forall t \geq t_{0},\left|x(t)-x^{*}\right| \leq L e^{-\eta\left(t-t_{0}\right)}$.

Note that exponential stability implies asymptotic stability which implies (local) stability.

\section{STABILITY ANALYSIS FOR TWO STRATE- GIES}

In this section we study the stability of ESS for the case of two strategies. Suppose that each individual of the population only uses a pure strategy. Denotes $\xi(t)=x_{1}(t)$ the 
proportion of individuals in the population using the first strategy at time $t$, the replicator dynamic of $\xi(t)$ is given by

$$
\begin{aligned}
\dot{\xi}(t) & =-K \cdot \delta \xi(t)(1-\xi(t)) \times \\
& {\left[\frac{\left(a_{12}-a_{11}\right)}{\delta} \xi\left(t-\tau_{1}\right)+\frac{\left(a_{21}-a_{22}\right)}{\delta} \xi\left(t-\tau_{2}\right)-\xi^{*}\right] }
\end{aligned}
$$

where $\delta=\left(a_{21}-a_{11}\right)+\left(a_{12}-a_{22}\right), \xi^{*}=\frac{a_{12}-a_{22}}{\delta}$. As in [34], if the payoff matrix A satisfies

$$
\left(a_{12}>a_{22}, a_{21}>a_{11}\right) \text { or }\left(a_{12}<a_{22}, a_{21}<a_{11}\right)
$$

then $0<\xi^{*}<1$ and $\xi^{*}$ is a unique interior stationary point so called fixed point, rest point or equilibrium point in $[17$, $33,24]$.

Now, we study the stability (2) at the interior equilibrium $\xi^{*}$.

\section{Linearization around the interior stationary point.}

Let $z(t)=\xi(t)-\xi^{*}$ then taking only the terms that are linear in $z$, equation (2) can be rewritten as

$$
\dot{z}(t)=-\gamma\left[\alpha z\left(t-\tau_{1}\right)+\beta z\left(t-\tau_{2}\right)\right]
$$

where $\gamma=K \xi^{*}\left(1-\xi^{*}\right), \alpha=a_{12}-a_{11}, \beta=a_{21}-a_{22}$. It is known $\left(\left[4\right.\right.$, pp.336],[15, pp.188]) that the steady state $\xi^{*}$ is asymptotically stable for (2) if the trivial solution of the linearized version (3) is asymptotically stable.

\section{Laplace transformation - Characteristic equation.}

Taking the Laplace transform of (3), we obtain the following characteristic equation

$$
s+\gamma\left[\alpha e^{-\tau_{1} s}+\beta e^{-\tau_{2} s}\right]=0
$$

\section{Stability condition.}

If all solutions of the characteristic equation (4) have a negative real part then the stationary point $z=0$ of (3) is (asymptotically) stable. Hence, the stationary point $\xi^{*}$ is asymptotically stable (2) . Conversely, if there is a solution $s$ of (4) with $\Re(s)>0$ then trivial solution is not stable for (3).

If $s=u+i v$ then equation (4) can be written as the system

$$
\left\{\begin{array}{c}
u+\alpha \gamma e^{-\tau_{1} u} \cos \left(\tau_{1} v\right)+\beta \gamma e^{-\tau_{2} u} \cos \left(\tau_{2} v\right)=0 \\
v-\gamma \alpha e^{-\tau_{1} u} \sin \left(\tau_{1} v\right)-\beta \gamma e^{-\tau_{2} u} \sin \left(\tau_{2} v\right)=0
\end{array}\right.
$$

\subsection{Symmetric delay}

Consider the following linear delay differential equation

$$
z(t)=-a z(t-\tau)
$$

with $\tau, a>0$ and its characteristic equation

$$
\lambda+a e^{-\lambda \tau}=0
$$

Necessary and sufficient condition of asymptotic stability as a function of the delay is given by the following lemma. A proof can be found in [15, proposition 1.2.8]

Lemma 1. A necessary and sufficient condition for all roots of (6) to have negative real parts is $2 a \tau<\pi$

When $\tau_{1}=\tau_{2}=\tau$, the trivial solution of (3) is asymptotically stable if

$$
K \tau<\frac{\delta \pi}{2 \delta_{1} \delta_{2}}
$$

and unstable if $K \tau>\frac{\delta \pi}{2 \delta_{1} \delta_{2}}$ where $\delta_{1}=a_{21}-a_{11}, \delta_{2}=$ $a_{12}-a_{22}$ and $\delta=\alpha+\beta \stackrel{2 \delta_{1} \delta_{2}}{=} \delta_{1}+\delta_{2}$.

The case $K \tau=\frac{\delta \pi}{2 \delta_{1} \delta_{2}}$ is called bifurcation point. By using lemma 1 , we conclude that the dynamic (2) is asymptotically stable at the stationary point $\xi^{*}=\frac{\delta_{2}}{\delta}$ if $K$ and $\tau$ satisfy (7).

\subsection{One strategy is not delayed}

If one of the strategies has no delay, the characteristic equation has the form

$$
\phi(s)=s+a+b e^{-s \tau}=0
$$

If $s=u+i v$ then (8) is equivalent to

$$
\left\{\begin{array}{c}
u+a=-b e^{-\tau u} \cos (\tau v) \\
v=b e^{-\tau u} \sin (\tau v)
\end{array}\right.
$$

Remark 1. If $(u, v)$ is a solution of (9) then

- $(u,-v)$ is also a solution of the system (9).

- One has $(u+a)^{2}+v^{2}=b^{2} e^{-2 \tau u}$.

Theorem 1 (see [11]). - If $|a| \neq|b|$. Then all roots $\lambda$ of the characteristic equation (8) have real part strictly negative if and only if

$$
|b|>|a| \text { and } \tau \leq \frac{\arcsin \left(\frac{1}{|b|} \sqrt{b^{2}-a^{2}}\right)}{\sqrt{b^{2}-a^{2}}}
$$

- If $a=b>0$ then characteristic equation has no roots with strictly positive real part.

Corollary 1. If the strategy 1 has no delay then

- If $|\beta|<|\alpha|$, increasing $\tau_{2}$ does not change the stability of the dynamical system (3). Thus the equation (2) is stable for any $\tau_{2} \geq 0$.

- If $|\beta|>|\alpha|$, the equation (2) is stable if $K \tau_{2}<\bar{\tau}_{2}$ and unstable if $K \tau_{2}>\bar{\tau}_{2}$ where

$$
\bar{\tau}_{2}:=\frac{\arcsin \left(\frac{1}{|\beta|} \sqrt{\beta^{2}-\alpha^{2}}\right)}{\xi^{*}\left(1-\xi^{*}\right) \sqrt{\beta^{2}-\alpha^{2}}}
$$

If the strategy 2 has no delay then

- If $|\beta|>|\alpha|$, increasing $\tau_{1}$ does not change the stability of the dynamical system (3). Thus the equation (2) is stable for any $\tau_{1} \geq 0$.

- If $|\beta|<|\alpha|$, the equation (2) is stable if $K \tau_{1}<\bar{\tau}_{1}$ and unstable if $K \tau_{1}>\bar{\tau}_{1}$ where

$$
\bar{\tau}_{1}:=\frac{\arcsin \left(\frac{1}{|\alpha|} \sqrt{\alpha^{2}-\beta^{2}}\right)}{\xi^{*}\left(1-\xi^{*}\right) \sqrt{\alpha^{2}-\beta^{2}}}
$$

Comparison between asymmetric/symmetric delay bound Let $\phi=\frac{\arccos (-a / b)}{\sqrt{b^{2}-a^{2}}}, b>|a|$ and $\Theta=\frac{\pi / 2}{a+b}$.

Case 1: $a>0$. Let $c=a / b$. One has $\frac{\arccos (-c)}{\sqrt{1-c^{2}}} \geq \frac{\pi / 2}{1+c}$. So, $\phi$ is greater than $\Theta$. Note that $\Theta$ is greater than the sufficient bound given in theorem 3 .

Case 2: $a<0$. So, $c<0$ and $\phi$ is lower than $\Theta$. 


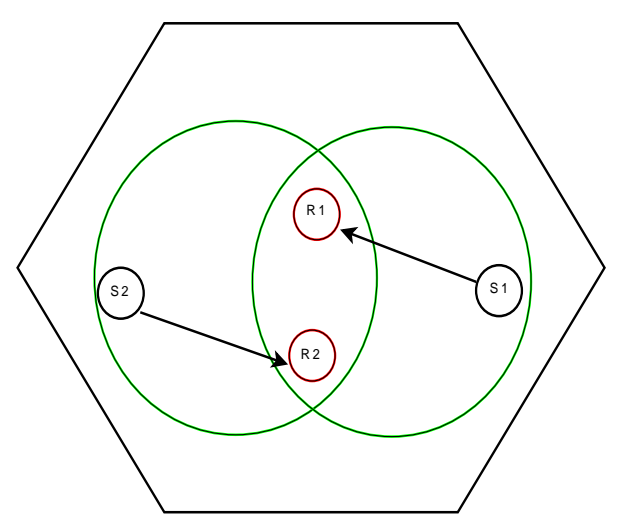

Figure 1: Interaction between S1 and S2.

\subsection{Others dynamics}

There are evolutionary scenarios which may not involve pairwise encounters between members of different populations. Fitness can be meaningful in determining the evolution of populations in context of genetic algorithms that involve interactions of a large number individuals. The replicator dynamics has been used for describing the evolution of road traffic congestion in which the fitness is determined by the strategies chosen by all drivers [25]. It has also been studied in the context of the association problem in wireless networks in [26].

On the other hand, there is a large number of population dynamics other than the replicator dynamics which have been used in the context of non-cooperative games. Examples are the Brown - von Neumann - Nash [7] dynamics, the fictitious play dynamics and gradient methods [23].

\section{EVOLUTIONARY MULTIPLE ACCESS GAME}

We consider a large population of players playing the Multiple Access Game introduced in [12]. Multiple Access Game introduces the problem of medium access. The Multiple Access Game is a symmetric nonzero-sum game, but the players have to share a common resource, the wireless medium, instead of providing it. Assume that the players use pure strategies. Consider two players Player I and Player II who want to send some packets to their receivers R1 and R2 using a shared medium. We assume that the players have a packet to send in each time slot and they can decide to transmit it or not. Suppose furthermore that Player I, Player II, R1 and $\mathrm{R} 2$ are in the power range of each other, hence their transmissions mutually interfere (see figure 1). Each of the players has two possible strategies: either transmit $(T)$ or to stay quiet $(S)$. If Player I transmits his packet, it incurs a transmission cost of $\Delta \in(0,1)$ after a delay $\tau_{T}$. The packet transmission is successful if Player II does not transmit (stays quiet) in that given time slot, otherwise there is a collision. If there is no collision, Player I gets a reward of 1 from the successful packet transmission after the delay $\tau_{T}$. We assume that the strategy stay quiet has no delay $\left(\tau_{S}=0\right)$.

The interaction is represented in figure 2 .

This matrix game has two pure Nash equilibria $(T, S)$ and $(S, T)$ a unique mixed Nash equilibrium (NE) given by (1$\Delta, \Delta)$. We shall show that this NE corresponds to an ESS

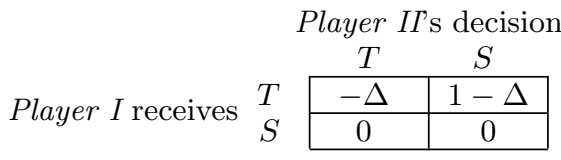

Figure 2: Multiple Access Game.

in the evolutionary game.

Note that the pure strategies $(T, S)$ and $(S, T)$ are also optimal in Pareto ${ }^{1}$ sense and non-symmetric. The scalar $1-\Delta$ (resp. $\Delta$ ) represents proportion of individuals which transmit (resp. stay quiet). When the two subpopulation use this strategy, they obtain the same fitness equal to zero.

Proposition 1. The strategy $(1-\Delta, \Delta)$ is the unique ESS of the evolutionary multiple access game.

Proof. The strategy $(1-\Delta, \Delta)$ is the unique symmetric Nash equilibrium. Thus, it is the only candidate to be ESS. Since, the strategies $T$ and $S$ have the same fitness when population profile is $(1-\Delta, \Delta)$. We check the condition of ESS given in definition 1 . Let

$$
A=K\left(\begin{array}{cc}
-\Delta & 1-\Delta \\
0 & 0
\end{array}\right)
$$

Then $\forall \xi \neq 1-\Delta$

$$
(1-\Delta-\xi, \xi-1+\Delta) A\left(\begin{array}{c}
\xi \\
1-\xi
\end{array}\right)=K(\xi-1+\Delta)^{2}>0
$$

We conclude that $(1-\Delta, \Delta)$ is an ESS.

The following proposition gives the stability of the interior equilibrium $(1-\Delta, \Delta)$ in the replicator dynamic (2).

Proposition 2. The mixed equilibrium $(1-\Delta, \Delta)$ is asymptotically stable if

$$
2 K \Delta(1-\Delta) \tau_{T}<\pi
$$

and not stable if $2 K \Delta(1-\Delta) \tau_{T}>\pi$.

Proof. We denote by $\xi(t)$ the proportion of individuals using the the strategy $T$ at time $t$. The replicator dynamic equation with delays in the Multiple Access Game becomes

$$
\dot{\xi}(t)=-K \cdot \xi(t)(1-\xi(t))\left[\xi\left(t-\tau_{T}\right)-1+\Delta\right]
$$

Taking linearized version of this equation and using lemma 1 , we obtain the results.

\subsection{Asymmetric positive delays}

Let

$$
\dot{z}(t)=-a z\left(t-\tau_{1}\right)-b z\left(t-\tau_{2}\right)
$$

where $a, b \in \mathbb{R}$ and $\tau_{1}, \tau_{2}$ are positive. The characteristic equation of 13 is given by

$$
s=-a e^{-\tau_{1} s}-b e^{-\tau_{2} s}
$$

Equation (14) can rewritten as

$$
\lambda=-e^{-r_{1} \lambda}-A e^{-r_{2} \lambda}
$$

where $\lambda=\frac{s}{a}, r_{1}=a \tau_{1}, r_{2}=a \tau_{2}$.

${ }^{1}$ An allocation of payoffs is said Pareto-optimal if the outcome cannot be improved upon without hurting at least one player. 
Gopalsamy [15] proved that when $a, b, \tau_{1}, \tau_{2}$ are positive, a sufficient condition for all roots of (14) to have negative real parts is $a \tau_{1}+b \tau_{2}<1$ and a necessary condition for the same is $a \tau_{1}+b \tau_{2}<\frac{\pi}{2}$. The following theorem give sufficient conditions of stability of (13) at zero.

Theorem 2 (see $[5,19]$ ). Suppose at least one of the following conditions holds

- $a+b>0,|a| \tau_{1}+|b| \tau_{2}<\frac{a+b}{|a|+|b|}$

- $a>0, a \tau_{1}<\frac{a-|b|}{a+|b|}$

- $b>0, b \tau_{2}<\frac{b-|a|}{|a|+b}$

Then equation (13) is exponentially stable.

Theorem 3. Suppose that $0<b<a$ and $\tau_{1}<\frac{1}{a+b}$. Then all roots of (14) have negative real parts (the equation (13) is asymptotically stable).

Proof. By hypothesis, one has $q:=\frac{b}{a} \in(0,1)$ and $r_{1}<$ $\frac{1}{1+q}$. Since all roots of equation (15) have negative real parts when $r_{1}=0$, if the conclusion fails, then there must be some $r_{1} \in\left(0, \frac{1}{1+q}\right]$ such that equation (15) has purely imaginary roots $\pm i y, y>0$ satisfying

$$
\begin{array}{r}
\cos \left(r_{1} y\right)=-q \cos \left(r_{2} y\right) \\
y-\sin \left(r_{1} y\right)=q \sin \left(r_{2} y\right)
\end{array}
$$

One has

$$
q^{2}-1=y^{2}-2 y \sin \left(r_{1} y\right)
$$

that is

$$
f(y):=\frac{y^{2}+1-q^{2}}{2 y}=\sin \left(r_{1} y\right)
$$

Since $-1 \leq \sin \left(r_{1} y\right) \leq 1 \Rightarrow y \in(1-q, 1+q)$.

$$
\begin{aligned}
f(y) & =\frac{y}{2}\left(1+\frac{1-q^{2}}{y^{2}}\right) \geq \frac{y}{2}\left(1+\frac{1-q^{2}}{(1+q)^{2}}\right) \\
& \geq y \frac{1}{1+q}>r_{1} y \geq \sin \left(r_{1} y\right)
\end{aligned}
$$

a contradiction with (16).

Corollary 2. If one of the following conditions holds, then $\xi^{*}$ is stable

- $\alpha+\beta=\delta>0, \quad K\left(|\alpha| \tau_{1}+|\beta| \tau_{2}\right)<\frac{\delta}{\xi^{*}\left(1-\xi^{*}\right)(|\alpha|+|\beta|)}$

- $\alpha>0, K \tau_{1}<\frac{\alpha-|\beta|}{\alpha \xi^{*}\left(1-\xi^{*}\right)(\alpha+|\beta|)}$.

- $\beta>0, K \tau_{2}<\frac{\beta-|\alpha|}{\beta \xi^{*}\left(1-\xi^{*}\right)(|\alpha|+\beta)}$.

- $0<\beta<\alpha, \quad K \tau_{1}<\frac{1}{\delta \xi^{*}\left(1-\xi^{*}\right)}$

\section{THE HAWK AND DOVE GAME}

We study in this section a very simple game known as Hawks and Doves. consider the problem of the Hawk-Dove game, analyzed by Smith and Price in [27]. In this game, two individuals compete for a resource. In biological contexts, the value of the resource corresponds to an increase in the fitness of the individual who obtains the resource. Each individual follows exactly one of two strategies described below:
- The Hawk is very aggressive and always fights for some resource. Fights between hawks are brutal and equal in fighting ability. Each hawk has a $50 \%$ chance of winning a Hawk-Hawk conflict.

- The Dove never fights for a resource - it displays in any conflict and if it is attacked it immediately withdraws before it gets injured.

These two simplified behavioral strategies employ very different means to obtain resources - fighting in Hawks and display in Dove. These differences in behavior have marked consequences on the chance of winning and of paying certain types of costs. The cost of the conflict reduces individual fitness by the value 2 . When a Hawk meets a Dove, the Dove immediately retreats and the Hawk obtains the resource value 1 and when two Doves meet, the resource is shared equally between them $\frac{1}{2}$. The fitness for the HawkDove game can be summarized according to the figure 3 .

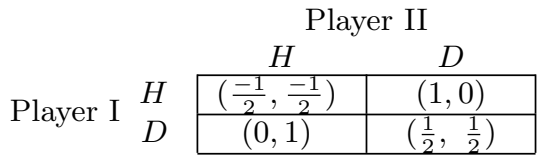

Figure 3: Strategic form representation of Hawk and Dove game. The numbers in parentheses represent the fitness accrued by players I and II, respectively for each possible strategy profile. The hawk is represented by $\mathrm{H}$ and the Dove by $\mathrm{D}$.

The matrix game given in figure 3 has two pure Nash equilibria $(H, D)$ and $(D, H)$ and a unique mixed Nash equilibrium given by $\left(\frac{1}{2}, \frac{1}{2}\right)$. The pure strategies profiles $(H, D)$ and $(D, H)$ are also Pareto optimal. The mixed Nash equilibrium $\left(\frac{1}{2}, \frac{1}{2}\right)$ is an ESS but is not a Pareto optimal strategy.

The replicator dynamic equation is given by

$$
\dot{\xi}(t)=-\frac{K}{2} \xi(t)(1-\xi(t))\left[3 \xi\left(t-\tau_{H}\right)-\xi\left(t-\tau_{D}\right)-1\right]
$$

$\xi^{*}=\frac{1}{2}$ is the unique interior stationary state of the equation (19). The mixed strategy $\left(\xi^{*}, 1-\xi^{*}\right)$ is asymptotically stable in the replicator dynamic without delay. In the dynamic (19),

- The strategy $\left(\xi^{*}, 1-\xi^{*}\right)$ is stable if $K \tau_{H}<4$. In particular if the strategy hawk has no delay then the interior stationary point is stable for any $\tau_{D}$.

- If the strategy Dove has no delay then the interior stationary point is stable when $K \tau_{H}<2 \sqrt{2} \arccos (-1 / 3) \approx$ 5.4040 and unstable if $K \tau_{H}>2 \sqrt{2} \arccos (-1 / 3)$.

\section{Numerical solutions.}

The numerical solutions given figure 4 are obtained when initial condition is 0.2 and delay $\tau_{H}$ between 0.2 and 7 . For the delay $\tau_{H}=7$ and $\tau_{S}=0.02$, (unstable situation) the proportion of hawks in population is negligible between 25 and 35. In this interval, we can consider that the opponent of the dove is also a dove. The hawks prefer to have today the half of the payoff by changing their strategy to Dove rather than to wait. The doves profit of these moments. We constat that for a large enough $\tau_{H}$ the solutions oscillate around the equilibrium point $1 / 2$. 


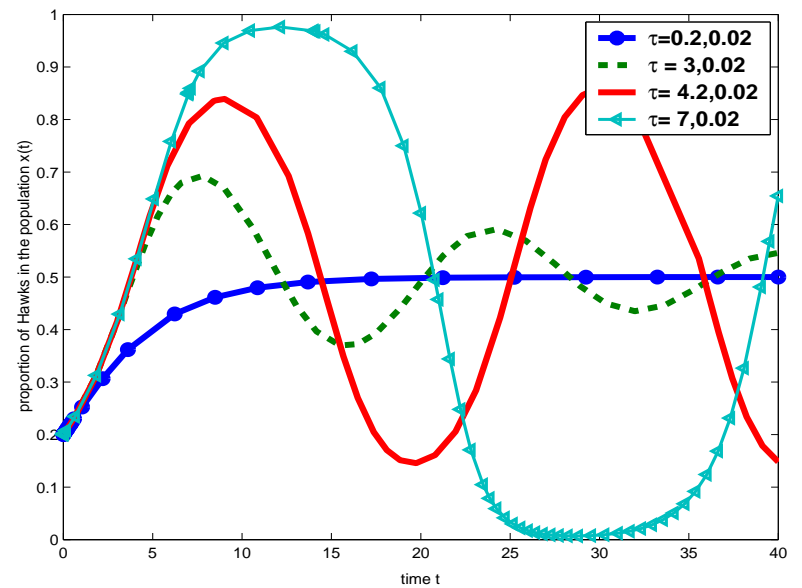

Figure 4: Oscillation for $\tau_{H}=7$ and convergence to the equilibrium point $1 / 2$ for $\tau_{H}=0.2$

\section{STABILITY ANALYSIS FOR GENERAL CASE}

In this section, we introduce replicator dynamics for a population with $n$ strategies and $n$ asymmetric delays with $n>2$.

$$
\left\{\begin{array}{c}
\dot{x}_{i}(t)=x_{i}(t)\left[e_{i} A x\left(t-\tau_{i}\right)-\sum_{k} x_{k}(t) e_{k} A x\left(t-\tau_{k}\right)\right] \\
i=1, \ldots, n .
\end{array}\right.
$$

Suppose that equation (6) has an interior stationary point $x^{*}$. The linearized equation is given by

$$
\begin{aligned}
\dot{z}_{i} & =x_{i}^{*}\left[e_{i} A z\left(t-\tau_{i}\right)-\sum_{k} x_{k}^{*} e_{k} A z\left(t-\tau_{k}\right)\right] \\
i & =1, \ldots, n
\end{aligned}
$$

where $z(t)=x(t)-x^{*}$. This equation can be written as

$$
\dot{z}(t)=K \sum_{l=1}^{n} B^{l} z\left(t-\tau_{l}\right)
$$

where $z(t)={ }^{t}\left(z_{1}(t), \ldots, z_{n}(t)\right)$ and $B^{l}=\left(b_{i k}^{l}\right) \in \mathbb{R}^{n \times n}$,

$$
b_{i k}^{l}=\left\{\begin{array}{cc}
x_{i}^{*}\left(1-x_{i}^{*}\right) a_{i k} & \text { if } l=i \\
-x_{i}^{*} x_{l}^{*} a_{l k} & \text { if } l \neq i .
\end{array}\right.
$$

Characteristic equation

$$
\operatorname{det}\left(\lambda I-K \sum_{l=1}^{n} B^{l} e^{-\tau_{l} \lambda}\right)=0 .
$$

A necessary and sufficient condition of stability of (20) is that all roots of the equation (21) have negative real parts.

\section{NYQUIST STABILITY CRITERION}

We use Nyquist stability criterion to determine numerically the stability of the non-linear systems (2). Nyquist plot is very used in automatic control for assessing the stability. It is represented by a graph in polar coordinates in which the gain and phase of a frequency response are plotted. This concept of plotting has been generalized to non-linear systems by Desoer, Stevens and Mason in [9, 21, 29]. Some applications of this notion of stability can be found in [32, $10,30,20]$.
Consider the complex equation $(*): 1+g(s)=0$ where $g$ is a meromorphic complex function. The Nyquist stability criterion of $(*)$ states that the number of unstable poles of $(*)$ is equal to the number of unstable poles of the function $g$ plus the number of encirclements of -1 of the Nyquist plot of the complex function $g$. This can be easily justified by applying Cauchy's principle of argument to the function $g(s)$.

$$
\frac{1}{2 i \pi} \oint_{C} \frac{g^{\prime}(s)}{g(s)} d s=Z-P
$$

where $C$ is simple closed contour, $Z$ is the number of zeros of $g$ contained in $C$ and $P$ the number of poles of $g$ inside the contour $C$ with $g$ having no zeros or poles on $C$.

\section{Characteristic equation: Nyquist plot approach.}

Nyquist plot of the function

$$
g: w \longmapsto \frac{\gamma \alpha e^{-i \tau_{1} w}+\gamma \beta e^{-i \tau_{2} w}}{i w}
$$

on $(0,+\infty)$ determine the stability of the dynamical system (3). For a population with $n$ strategies, the function $g$ is given by

$$
g: \lambda \mapsto \operatorname{det}\left(I-\frac{K}{\lambda} \sum_{l=1}^{n} B^{l} e^{-\tau_{l} \lambda}\right)-1
$$

We keep the same parameters as in the Hawk and Dove game given in section 5 and we discuss about stability by varying delays in figure 5,7 and 6 . In the figure 7 , delays are $\tau_{H}=7, \tau_{D}=0.2$, the graph encircles the point $-1+0 \times i$ i.e the system (3) is not stable. In figure 5 and 6 , delays are respectively $\tau_{H}=\tau_{D}=0.2$, and $\tau_{H}=0.2, \tau_{D}=10$ respectively and the system is stable. In all these figures, $K=1$. All turn out to confirm the stability condition that we obtained in previous sections.

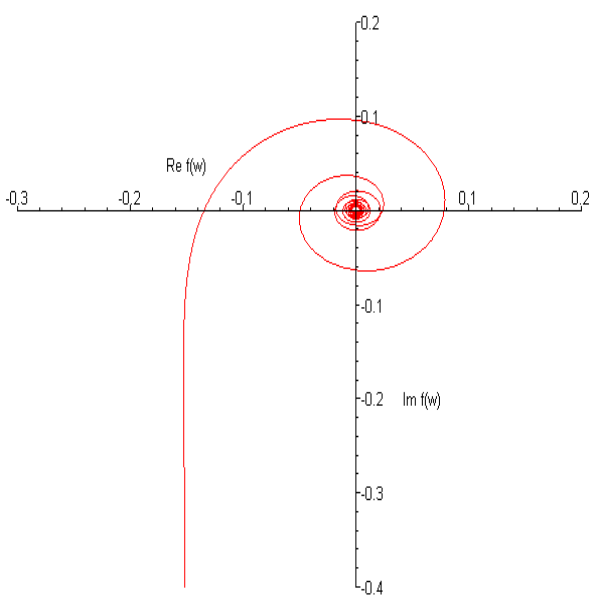

Figure 5: Stable situation: $\tau_{H}=0.2$

\section{CONCLUSIONS}

Delay is very important in evolutionary game dynamics. In this paper, we considered evolutionary games with one population of users and studied asymmetric delays impact on convergence to ESS. We showed that the evolutionary 


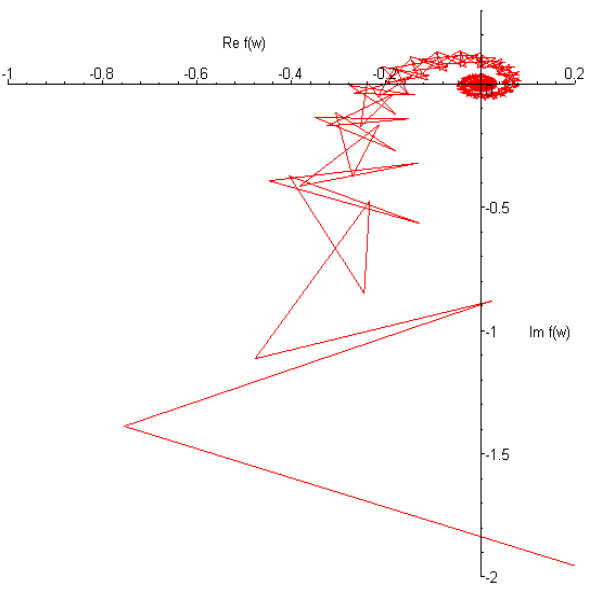

Figure 6: Stable situation $\tau_{H}=0.2, \tau_{D}=10$, increasing $\tau_{D}$ does not change the stability

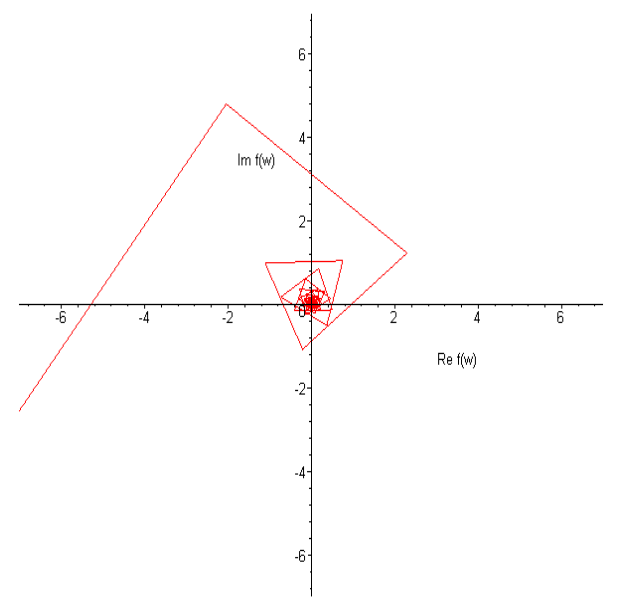

Figure 7: Unstable situation $\tau_{H}=7$

multiple access game has an unique ESS which can be unstable when the delay of transmission is large.

\section{Acknowledgement}

This work was supported by the Bionets European Project.

\section{REFERENCES}

[1] J. Alboszta and J. Miękisz, Stability and evolutionary stable strategies in discrete replicator dynamics with delay, J. Theor. Biology, 2004.

[2] E. Altman and T. Başar and N. Malouch, The role of information update in flow control, Netw., 2004.

[3] J. Arino, L. Wang and G. Wolkowicz, An Alternate Formulation for a Delayed Logistic Equation, Journal of Theoretical Biology,2006,241,1.

[4] R. Bellman and K.L. Cooke, Differential difference equations, Academic Press, New York, 1963.

[5] L. Berezansky and E. Braverman, On stability of some linear and nonlinear delay differential equations, J. Math. Anal. Appl., Vol. 314, No. 2 (2006), pages 391-411.

[6] N. Bonneau,E. Altman, M. Debbah, and G. Caire, An Evolutionary Game Perspective to ALOHA with Power Control, 19th International Teletraffic Congress, Beijing, China, Aug. 29-Sep.,2005.
[7] G. Brown and J. Von Neumann, Solutions of games by differential equations, In H. Kuhn and A. Tucker, editors, Contributions to the Theory of Games I, Annals of Mathematics Studies 24, pages 73-79. Princeton University Press, 1950.

[8] R. Cressman, Evolutionary Dynamics and Extensive Form Games, MIT Press, Cambridge,2003.

[9] C. A. Desoer and Yung-Terng Wang, On the Generalized Nyquist Stability Criterion, IEEE Transactions on Automatic Control, April 1980, vol. AC-25, NO. 2,187.

[10] N. Dukkipati and N. McKeown, Stability Analysis of RCP for Long-lived Flows, fev 2004, available at http://yuba.stanford.edu/ñanditad/GroupMeetFeb04.pdf.

11] H. I. Freedman and Y. Kuang, Stability Switches in Linear Scalar Neutral Delay Equations, Funkcialaj Ekvacioj, 34 (1991) 187-209.

[12] M. Felegyhazi and J.-P. Hubaux, Game Theory in Wireless Networks: A Tutorial, EPFL technical report, LCA-REPORT-2006-002, February,2006.

[13] Daniel Friedman, On economic applications of evolutionary game theory, Journal of Evolutionary Economics, 1998,8,1,15-43.

[14] H. Gintis, Game Theory Evolving, Princeton University Press, 2000.

[15] K. Gopalsamy, Stability and Oscillation in Delay Differential Equations of Population Dynamics, Kluwer Academic Publishers, London,1992.

[16] J. Hofbauer and K. Sigmund, Evolutionary game dynamics, American Mathematical Society, Vol 40 No. 4, pp. 479-519,2003.

[17] J. Hofbauer and K. Sigmund, Evolutionary Games and Population Dynamics, Cambridge University Press, Cambridge, UK, 1998.

[18] R. Johari and D. Tan,End-to-End Congestion Control for the Internet: Delays and Stability, IEEE/ACM Transactions on Networking,2001,9,6.

[19] X. Li and S. Ruin and J. Wei, Stability and bifurcation in delay-differential equations with two delays, Journal of math. analysis and applications, vol. 236, no2, pp. 254-280, 1999.

[20] S. Liu, T. Başar and R. Srikant, Exponential-RED: A Stabilizing AQM Scheme for Low- and High-Speed TCP Protocols , IEEE/ACM Trans. on Networking, vol. 13, no. 5,oct., 2005.

[21] S.J. Mason, A comment on Dr Vazsonyi's Paper: "A generalized Nyquist's stability criteria", May,20, Journal of Applied Physics - September 1949 - Volume 20, Issue 9 , p. 867.

[22] F.Y. Ren and C. Lin and B. Wei, A Nonlinear Control Theoretic Analysis to TCP-RED System, Computer Networks ,vol.49: 580-592, 2005.

[23] J. B. Rosen, Existence and uniqueness of equilibrium points for concave N-person games, Econometrica 33, 520-534, 1965.

[24] L. Samuelson, Evolutionary Games and Equilibrium Selection, MIT Press,1997.

[25] W.H. Sandholm, Potential games with continuous player sets, Journal of Economic Theory, 97:81-108,2001.

[26] S. Shakkottai, E. Altman and A. Kumar, The Case for Non-cooperative Multihoming of Users to Access Points in IEEE 802.11 WLANs, IEEE Infocom,2006.

[27] J. M. Smith and GR Price, The logic of animal conflict, Nature 246,15-18,1973.

[28] J. M. Smith, Evolution and the theory of Games, Cambridge University Press, 1982.

[29] P. K. Stevens, A Generalization of the Nyquist Stability Criterion, IEEE Transactions on Automatic Control, vol AC-26, no. 3, june ,1981.

[30] Deb Supratim and R. Srikant, Global Stability of congestion controllers for the internet, Proc. of IEEE Conference on Decision and Control, Las Vegas, Dec, 
2002.

[31] P. Taylor and L. Jonker, Evolutionary Stable Strategies and Game Dynamics, Mathematical Biosciences, 1978, $16,76-83$.

[32] G. Vinnicombe, On the stability of networks operating TCP-like congestion control, In 15th IFAC World Congress on Automatic Control, July 2002.

[33] J.W. Weibull, Evolutionary Game Theory, Cambridge, MA: MIT Press,1995.

[34] T. Yi and Z. Wang, Effect of Time Delay and Evolutionary Stable Strategy, J. theor. Biol, 187, 111-116, 1997.

[35] Y. Zheng and Z. Feng, Evolutionary game and resources competition in the Internet, Modern Communication Technologies, SIBCOM-2001, 2001. 